\title{
Role of PD-1/PD-L1 inhibitors in the treatment of recurrent/metastatic head and neck squamous cell carcinoma: a systematic review and meta-analysis
}

\author{
gaofei Yin ${ }^{1}$, Wei Guo ${ }^{1}$, Hanyuan Duan ${ }^{1}$, and zhigang Huang ${ }^{1}$ \\ ${ }^{1}$ Beijing Tongren Hospital Department of Otorhinolaryngology and Head and Neck Surgery
}

April 29, 2020

\begin{abstract}
Background: The use of immunotherapy to treat recurrent/metastatic squamous cell carcinoma of the head and neck has become a popular research topic in recent years, and many clinical trials have been carried out. Objectives: To systematically evaluate the efficacy and safety of PD-1/PD-L1 inhibitors in the treatment of recurrent/metastatic head and neck squamous cell carcinoma. Methods: We searched PubMed, Embase, Cochrane Library and other databases up to 1 November 2019 for publications reporting the use of PD-1/PD-L1 inhibitors in the treatment of squamous cell carcinoma of the head and neck. Revman 5.0 was used for combination analysis, and the overall survival (OS), progression-free survival (PFS), overall response rate (ORR), and adverse events were determined. Results: Five articles were included. Compared with other treatment methods, this meta-analysis showed that treatment with PD-1/PD-L1 inhibitors can significantly improve OS $(\mathrm{P}<0.0001)$, but there was no significant improvement in PFS or ORR. The risk of anaemia and nausea was significantly reduced by treatment with PD-1/PD-L1 inhibitors. Conclusions and Significance: Treatment with PD-1/PD-L1 inhibitors alone can improve the overall survival rate for recurrent/metastatic squamous cell carcinoma of the head and neck but there is no obvious advantage in other aspects and adverse events.
\end{abstract}

\section{Main text introduction}

Squamous cell carcinoma of the head and neck (HNSCC) is the most common malignant tumour in that region. According to the latest GLOBOCAN report [1], HNSCC is the ninth most common malignant tumour in the world. In 2018, the number of new cases worldwide was 843860 , accounting for $4.6 \%$ of all new cancer cases. Because of the nature of this disease, there are a large number of patients who are at the late stage or local late stage at the time of treatment. The 5-year survival rate of these patients with traditional treatment is only $40 \%$ [2], and the treatment of patients with recurrent/distant metastasis is more difficult. At present, the first-line treatment for advanced HNSCC is cetuximab combined with cisplatin and 5 -FU, but there are low response rates combined with drug resistance [3].

With the anti-tumour effect of immunotherapy in non-small cell lung cancer and other tumours, the study of immunosuppressive checkpoint inhibitors in HNSCC has become a popular research topic at the present time. In addition, Nivolumab [4] and Pembrolizumab [5] have been approved by the FDA to be used in the treatment of recurrent or metastatic HNSCC in the event of platinum treatment failure. There are a growing number of corresponding clinical trials, but there has been no summary analysis showing the efficacy of programmed cell death protein 1 (PD-1) and programmed cell death ligand protein 1 (PD-L1) inhibitors.

This study aimed to evaluate treatment of HNSCC with PD-1/PD-L1 inhibitors through a meta-analysis of all previous randomized controlled trials to form a preliminary reference for follow-up clinical trials and clinical treatment. 


\section{Materials and methods}

Search strategy

Two authors conducted the search and study review independently. We searched PubMed, Embase, Cochrane Library, CVID, CBM, CNKI and Web of Science up to 1 November 2019. The search terms used were: PD1/PD-L1 inhibitors or Pembrolizumab or Keytruda or Nivolumab or Opdivo or Durvalumab or MEDI4736 or Atezolizumab or checkpoint inhibitors and head and neck cancer or HNC or head and neck squamous cell carcinoma or head and neck neoplasms or HNSCC. We also searched 'clinicaltrials.gov' with the search term Head and Neck Squamous Cell Carcinoma, and we also searched for Squamous cell carcinoma of head and neck, Squamous cell carcinoma of the head and neck, head and neck cancer, head and neck neoplasms or HNSCC and PD-1/PD-L1 inhibitors or Pembrolizumab or Keytruda or Nivolumab or Opdivo or Durvalumab or MEDI4736 or Atezolizumab or checkpoint inhibitors.

Study selection

Randomized controlled trials (RCT) were used, and the inclusion criteria for our meta-analysis were as follows: 1) single drug treatment with PD-1/PD-L1 inhibitors; 2) the papers should have exact data whether in the time domain or frequency domain; 3) the groups being compared were treated with chemotherapy, targeted therapy, a combination of two immunosuppressive inhibitors or other drug combinations. Exclusion criteria for the meta-analysis were as follows: 1) non-randomized trials; 2) studies that were not published in English or Chinese; 3) studies that did not report the end points of overall response rate (ORR), overall survival (OS), and progression-free survival (PFS).

Data extraction and quality assessment

Two authors independently examined all potentially relevant studies identified through the search. We read all of the papers to determine whether they met our inclusion criteria and decided whether they should be included. Any disagreements were resolved by consensus. Data were extracted by one researcher and supervised by another researcher and included: 1) author information; 2) publication year; 3) number of patients in the study; 4) treatment plan; 5) study design; 6) the main results.

Data synthesis and statistical analysis

We used Review Manager software (version 5.0; Cochrane Collaboration, London, UK) as the tool for calculating the outcomes. The relative risk (RR) and 95\% confidence interval (CI) were used to compare the outcomes between the different groups. The chi-squared test was used to test heterogeneity. Cochran's $\mathrm{Q}$ test was applied to assess the heterogeneity of the different studies. The inconsistency of each study was measured with the I2 statistic. When I2 [?] 50\%, a random effects model was used to take account of clinical heterogeneity in the data, otherwise a fixed effects model was used. Sensitivity analysis was used to analyze the effect of poor quality studies, and publication bias was analysed using an inverted funnel chart test.

\section{Results}

Description of the studies

In total, 1540 articles were initially identified and five were finally selected according to the exclusion criteria, with a total of 2005 patients The specific screening process is shown in Figure 1. The five articles included four phase III RCT clinical trials and one phase II RCT clinical trial. Treatment with PD-1/PD-L1 inhibitors was one of the intervention programmes, and this was compared with other treatment methods. Overall response rate (ORR), overall survival (OS), and progression-free survival (PFS) were the end points in each study. The median follow-up time ranged from 5.2 to 17 months. Specific information on each study is shown in Table 1 and the results of methodological quality evaluation for each study are shown in Table 2 .

[Figure 1 near here]

Outcome results of meta-analysis 
Overall response rate (ORR): All five of the articles reported the ORR of the patients in each group. Through software analysis (Figure 2), there was heterogeneity between the studies (I2 $=87 \%$ ), so using the random effects model, the results showed that there was no statistically significant difference between the group treated with PD-1/PD-L1 and the group with other treatments $(\mathrm{P}=0.35)$.

[Figure 2 near here]

Overall survival (OS): Four of the five articles reported the OS in each experimental group. Through software analysis (Fig. 3), there was no heterogeneity $(\mathrm{I} 2=0 \%$ ), so the fixed effects model was used. The results showed that treatment with PD-1/PD-L1 inhibitors alone could reduce the risk of death in patients with recurrent/metastatic HNSCC, and the difference between patients treated with PD-1/PD-L1 and the group with other treatments was statistically significant $(\mathrm{P}=0.004)$.

[Figure 3 near here]

Progression-free survival (PFS): Four of the five articles reported the PFS of patients in each group. Through software analysis (Figure 4), there was no heterogeneity $(\mathrm{I} 2=0 \%)$, so the fixed effects model was used. The results showed that there was no statistically significant difference in PFS between the group treated with $\mathrm{PD}-1 / \mathrm{PD}-\mathrm{L} 1$ and the group with other treatments $(\mathrm{P}=0.47)$.

[Figure 4 near here]

Adverse events

The five articles included in the study reported the adverse events of diarrhoea, asthenia, rash, fatigue, anaemia and nausea after treatment. Meta-analysis showed that there was no significant difference in diarrhoea, asthenia, rash or fatigue between the PD-1/PD-L1 inhibitor treatment group and the other treatment group, but $\mathrm{PD}-1 / \mathrm{PD}-\mathrm{L} 1$ inhibitor treatment significantly reduced the risk of anaemia $(\mathrm{P}<0.0001)$ and nausea $(\mathrm{P}<0.0001)$, as shown in Table 3.

Publication bias analysis

A funnel chart test was carried out for OS, PFS, ORR and adverse reaction results to judge the effect of possible publication bias. However, due to the inclusion of less than 10 documents, it was impossible to use a forest map to judge publication bias. In order to reduce the effects of publication bias, we searched the unpublished clinical trial registry data. We use search terms Head and Neck Squamous Cell Carcinoma, and also searched for Squamous cell carcinoma of head and neck, Squamous cell carcinoma of the head and neck, head and neck cancer, head and neck neoplasms or HNSCC and PD-1/PD-L1 inhibitors or Pembrolizumab or Keytruda or Nivolumab or Opdivo or Durvalumab or MEDI4736 or Atezolizumab or checkpoint inhibitors. We found 217 studies but after removal of duplicates and consideration of inclusion criteria, we had to remove them all.

\section{Discussion}

In recent years, with the development of immunotherapy, there has been an increasing number of immunotherapy studies on HNSCC, and there are also drugs approved for treatment of recurrent/metastatic HNSCC after platinum treatment failure. The two trials involved in this paper, checkmate-141 [8] and keynote-012 [7], are the basis for the approval of nivolumab and pembrolizumab, both of which are PD-1 inhibitors. PD-1 is a programmed death protein, which is an important immune checkpoint for tumours with which they can evade immune surveillance. It is expressed on the surface of activated T cells, B cells and NK cells [11]. Like many solid tumours, HNSCC can evade the immune response of host cells by expressing immunosuppressive ligands. For example, PD-L1 has two major ligands, PD-L1/PD-L2, which exist in tumour cells, antigen-presenting cells and stromal cells. The tumour microenvironment induces infiltration of $\mathrm{T}$ cells with a high expression of PD-1 and tumour cells with a high expression of PD-L1/PD-L2. The combination of the two leads to the continuous activation of the PD-1 pathway, the down-regulation of $\mathrm{T}$ cell function and inhibition of the anti-tumour effect of the immune system. At present, the main antibodies against PD-1 
are nivolumab and pembrolizumab, and the main antibodies against PD-L1 are atezolizumab, avelumab and durvalumab.

Of the five articles, three were PD-1/PD-L1 inhibitor single drug treatments compared with chemotherapy. The first article was a PD-L1 inhibitor single drug treatment compared with CTLA-4 inhibitor; the second was a PD-L1 inhibitor single drug treatment compared with PD-L1 inhibitor combined with CTLA-4 inhibitor; the third article was a PD-L1 inhibitor single drug treatment compared with chemotherapy and a PD-1 inhibitor treatment combined with chemotherapy. Meta-analysis results showed that there were differences in OS among the groups, but no differences in PFS and ORR. In the article by Burtness et al. [10], the ORR was $17 \%$ in the study group compared with $36 \%$ in the control EXTREME group. Although immunotherapy is a promising treatment, its efficacy in patients is limited. The effective rate of single drug treatment in patients with HNSCC was only about $20 \%$. The effective rates in the five articles included in this paper were $14.6 \%, 13.3 \%, 26.1 \%, 9.2 \%$ and $17 \%$. The median survival times in the five articles were 8.4 months (6.4-9.4), 7.5 months (5.5-9.1), 9.5 months (9.1-NR), 6.0 months (4.0-11.3), and 14.9 months (no range reported) in the groups with PD-L1 inhibitor single drug treatment. Apart from one article, the median survival time was higher than that of the control group, including the standard chemotherapy, single treatment with CTLA-4 inhibitor, but lower than that of PD-1 inhibitor combined with CTLA-4 inhibitor (6.0 vs 7.6 months).

The use of immunotherapy combined with other adjuvant therapies has been increasing in recent years. For example, the sensitization effect and mechanism of action of chemotherapy and radiotherapy are all supported by the literature. Therefore, it is also hoped that the response rate can be improved by combining immunotherapy with other therapies to further extend survival time. In recent and ongoing clinical trials, in addition to the use of PD-1/PD-L1 inhibitors as a single drug for treatment, the trials include their combination with other immunosuppressants, chemotherapy and radiotherapy, etc. Table 4 shows the current stages of the ongoing phase III clinical trials (registered at clinicaltrials.gov) for immunotherapy of HNSCC. In addition, a clinical trial of neoadjuvant immunotherapy for patients with locally advanced or operable HNSCC is also being carried out, combined with radiotherapy and chemotherapy to regulate the immune environment and to work alongside immunotherapy to improve the therapeutic effect [12]. These trials are expected to provide high-level clinical evidence to support the management of future therapy.

Unlike other treatments, there are many immune-related adverse events associated with immunotherapy such as anaemia, renal failure, pneumonia, etc. [13]. In the five articles included in this paper, four of them recorded adverse events including diarrhoea, asthenia, rash, fatigue, anaemia, nausea, etc. Our metaanalysis showed that PD-1/PD-L1 inhibitors did not increase the risk of adverse events, but in fact, reduced the risk of anaemia and gastrointestinal reactions. The main reason may be because the control group was mainly composed of chemotherapy or chemotherapy-based comprehensive treatments. No matter what kind of treatment, the incidence of adverse events in patients was more than $10 \%$. In the articles included in this study, the adverse events caused by immunotherapy included skin reaction, endocrine, gastroenteric, therapeutic, pulmonary, infection reaction, renal injury, hypothyroidism, and pneumatic events. In the four articles included in this study and mentioning adverse reactions, the incidences of adverse reactions in the PD-1/PD-L1 treatment groups were higher than those in the immunotherapy combined with other auxiliary treatment groups ( $26 \%$ vs $12 \% ; 63.1 \%$ vs $55.4 \% / 57.9 \% ; 58.9 \%$ vs $37.5 \% ; 26 \%$ vs $12 \%$ ). Therefore, from the point of view of adverse reactions, combination therapy may improve the response rate and improve the therapeutic effect. There are also no increased risks from the point of view of side effects. It also provides help for the formulation of future treatment plans and the improvement of intolerable treatments.

\section{Conclusion}

This study shows that, based on the current evidence, PD-1/PD-L1 inhibitors alone can improve the OS of patients with recurrent/metastatic head and neck squamous cell carcinoma, without serious adverse reactions, but the ORR still needs to be improved. In future research, the combination of drugs and new adjuvant treatments will help immunotherapy play a better role in the treatment of HNSCC. 


\section{Methodological considerations / limitations:}

The number of studies included in this paper is limited, and the conclusions need to be verified by a larger number of articles.

\section{References}

[1] Bray F, Ferlay J, Soerjomataram I, Siegel RL, Torre LA, Jemal A. Global cancer statistics 2018: GLOBOCAN estimates of incidence and mortality worldwide for 36 cancers in 185 countries. CA Cancer J Clin. 2018;68(6):394-424.

[2] Winquist E, Agbassi C, Meyers BM, et al. Systemic therapy in the curative treatment of head and neck squamous cell cancer: a systematic review. J Otolaryngol Head Neck Surg. 2017;46(1):29.

[3] Lydiatt W, O'Sullivan B, Patel S. Major changes in head and neck staging for 2018. Am Soc Clin Oncol Educ Book. 2018;38:500-514.

[4] Gillison ML, Blumenschein G Jr, Fayette J, et al. CheckMate 141: 1-year update and subgroup analysis of nivolumab as first-line therapy in patients with recurrent/metastatic head and neck cancer. Oncologist. 2018;23(9):1079-1082.

[5] Larkins E, Blumenthal GM, Yuan W, et al. FDA Approval Summary: Pembrolizumab for the treatment of recurrent or metastatic head and neck squamous cell carcinoma with disease progression on or after platinum-containing chemotherapy. Oncologist. 2017;22(7):873-878.

[6] Cohen EEW, Soulières D, Le Tourneau C, et al. Pembrolizumab versus methotrexate, docetaxel, or cetuximab for recurrent or metastatic head-and-neck squamous cell carcinoma (KEYNOTE-040): a randomised, open-label, phase 3 study. Lancet. 2019;393(10167):156-167.

[7] Ferris RL, Blumenschein G Jr, Fayette J, et al. Nivolumab for recurrent squamous-cell carcinoma of the head and neck. N Engl J Med. 2016;375(19):1856-1867.

[8] Kiyota N, Hasegawa Y, Takahashi S, et al. A randomized, open-label, Phase III clinical trial of nivolumab vs. therapy of investigator's choice in recurrent squamous cell carcinoma of the head and neck: A subanalysis of Asian patients versus the global population in Checkmate 141. Oral Oncol. 2017;73:138-146.

[9] Siu LL, Even C, Mesía R, et al. Safety and efficacy of durvalumab with or without tremelimumab in patients with PD-L1-low/negative recurrent or metastatic HNSCC: The Phase 2 CONDOR randomized clinical trial. JAMA Oncol. 2019;5(2):195-203.

[10] Burtness BA, Harrington KJ, Greil R, Soulieres D. KEYNOTE-048: Phase III study of first-line pembrolizumab (P) for recurrent/metastatic head and neck squamous cell carcinoma (R/M HNSCC). Ann Oncol. 2018;29(suppl_8).

[11] Pardoll DM. The blockade of immune checkpoints in cancer immunotherapy. Nat Rev Cancer. 2012;12(4):252264 .

[12] Bauman JE, Cohen E, Ferris RL, Adelstein DJ, Brizel DM, Ridge JA, et al. Immunotherapy of head and neck cancer: emerging clinical trials from a National Cancer Institute Head and Neck Cancer Steering Committee Planning Meeting. Cancer. 2017;123:1259-1271.

[13] McKay RR, Martini D, Moreira RB, Hamieh L, Norton C, Mullane SA, et al. Outcomes of PD-1/PD-L1 responders who discontinue therapy for immune-related adverse events (irAEs): results of a cohort of patients (pts) with metastatic renal cell carcinoma (mRCC). J Clin Oncol. 2017;35(suppl 6S):abstr 467.

\section{Figure captions}

Figure 1. Flow chart of the search strategy.

Figure 2. PD-1/PD-L1 inhibitors vs control treatment in overall response rate (ORR). 
Figure 3. PD-1/PD-L1 inhibitors vs control treatment in overall survival (OS).

Figure 4. PD-1/PD-L1 inhibitors vs control treatment in progression-free survival (PFS).

\section{Hosted file}

Table 1-meta.docx available at https://authorea.com/users/316464/articles/446605-role-of-pd1-pd-11-inhibitors-in-the-treatment-of-recurrent-metastatic-head-and-neck-squamous-cell-carcinomaa-systematic-review-and-meta-analysis

\section{Hosted file}

Table 2-meta.docx available at https://authorea.com/users/316464/articles/446605-role-of-pd1-pd-11-inhibitors-in-the-treatment-of-recurrent-metastatic-head-and-neck-squamous-cell-carcinomaa-systematic-review-and-meta-analysis

\section{Hosted file}

Table 3-meta.docx available at https://authorea.com/users/316464/articles/446605-role-of-pd1-pd-11-inhibitors-in-the-treatment-of-recurrent-metastatic-head-and-neck-squamous-cell-carcinomaa-systematic-review-and-meta-analysis
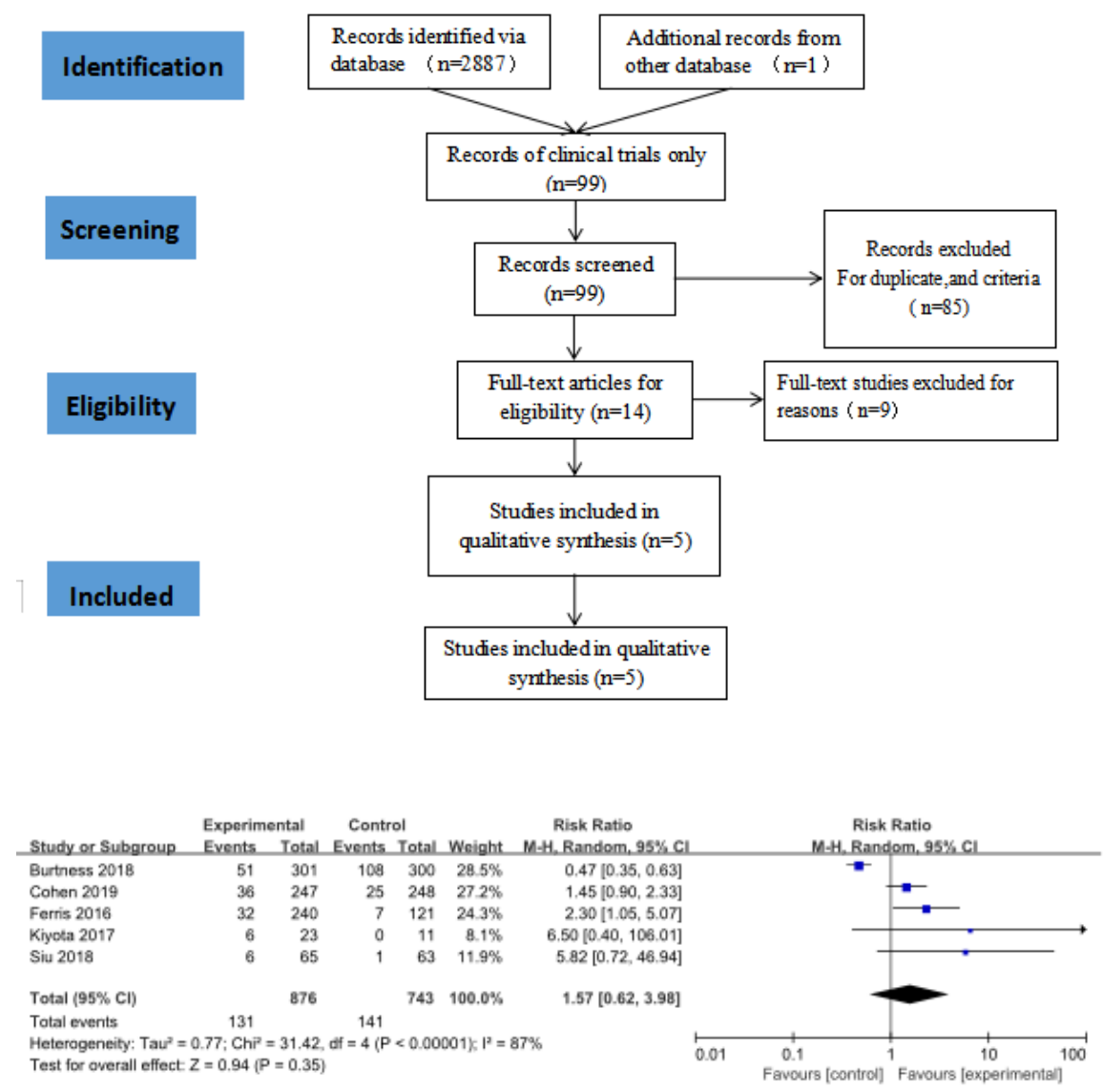


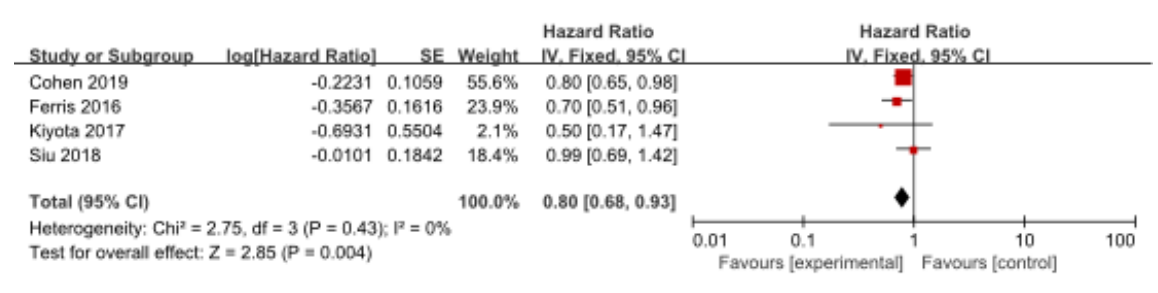

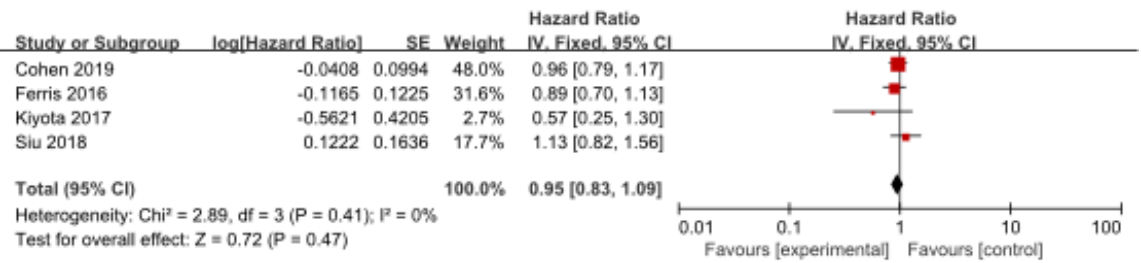

